\title{
Dynamic Resource Provisioning with Authentication in Distributed Database
}

\author{
Anju Aravind K \\ Shree Venkateshwara Hi-Tech \\ Engineering College \\ Gobi, India
}

\author{
Dr. T. Senthil Prakash \\ Shree Venkateshwara Hi-Tech \\ Engineering College \\ Gobi, India
}

\author{
M. Rajesh \\ Shree Venkateshwara Hi-Tech \\ Engineering College \\ Gobi, India
}

\begin{abstract}
Data center have the largest consumption amounts of energy in sharing the power. The public cloud workloads of different priorities and performance requirements of various applications ${ }^{[4]}$. Cloud data center have capable of sensing an opportunity to present different programs. In my proposed construction and the name of the security level of imperturbable privacy leakage rarely distributed cloud system to deal with the persistent characteristics there is a substantial increases and information that can be used to augment the profit, retrenchment overhead or both. Data Mining Analysis of data from different perspectives and summarizing it into useful information is a process. Three empirical algorithms have been proposed assignments estimate the ratios are dissected theoretically and compared using real Internet latency data recital of testing methods.
\end{abstract}

Keywords: Mining, MD5, green computing, workload imitation, power consumption

\section{INTRODUCTION}

Most data centers today have a three or four tier hierarchical networking structure. Three tier network architectures were designed around client-server applications and single-purpose of application server. Client and server applications are caused traffic to flow primarily in patterns: from a server up to the data center core, to the environment core where it moves out to the internet. These large core switches usually contain the vast majority of the intelligence in the network. The cost of builds and operates the large computer platform and a focus on service quality and cost-efficient driving will require cost estimation and on the capacity of processing and storage.

The cloud consists of

Private Cloud: The infrastructure is provisioned for exclusive use by a single organization.

Public Cloud: The infrastructure is provisioned for open use by the general public.

Hybrid Cloud: There is a system infrastructure of two or more distinct cloud Infrastructures (private, community, or public) that remain unique entities, but are bound together by standardized or proprietary technology that enables data and application portability.

Community Cloud: Consumers who share the concerns of the infrastructure for personal use by the provision of a particular community.

While Cloud computing is not equivalent to virtualization, virtualization technology is heavily used to operate a cloud environment system. In the virtualization have one of the host that ran a single operating system now has the ability to run multiple guest operating systems as virtual machines [VMs]. The VMs are created for fast and easily data storage in a cloud environment. The infrastructure environment is invisible for abstracted from the consumer. The computing of firmware system to provide virtual machine and it allows to operating directly on underlying hardware but within specified constraint. It is the software that manages communications between the physical server memory and CPU or processing capability and the virtual machines that are running. The software allows VMs to be quickly provisioned or decommissioned. So the data center contains delay process, security process, mining process and also cost efficiency.

First, in data centre have different perspectives and useful information of data mining [DM]. In DM information that can be used to increase the revenue and it is the process of summarizing the costs or both are reduced. It allows the user to analyze the data with different dimensions or angles. To categorize and summarize the relationships are identified the data. The proposed method of Support Vector Machine for data mining system [DMS] is designed to take the advantage of powerful processors and shared pools to the calculation is performed using the message passing paradigm for data that is distributed across processors. The calculation results are then collected, and the process is repeated with new data processor.

Second, data center security concerns to secure all aspects of cloud data ${ }^{[2]}$. Many of these features are not unique in cloud system: Irrespective of the data stored on it is vulnerable attack. Therefore the cloud computing have security, access control, malware protection structures, reducing attack surfaces, safety design and implementation of all data including computing security. In this proposed method having MD5 is used to secure the data in data center.

Third, resource provisioning in cloud computing over the last few years, has emerged as new computing model to allowing utility based delivery of services to end users ${ }^{[1]}$. Cloud computing relies on virtualization technologies to provide ondemand resources according to the end user needs but problems with resource allocation pose an issue for the management of several data centers. In my proposed gossip protocol of green computing based virtualization can be used to increase energy efficiency in substrate networks, allowing consolidation through the virtual hosting of different resources on the same substrate resource. Migrating resources virtually allows the network to balance its overall energy load and reduce the total power consumption of the data center. The dynamic provisioning concept is used to allocate the resources dynamically. 


\section{RELATED WORK}

\subsection{Datacenter}

The datacenter is the collection of servers, the cloud computing and information technology between physical servers to migrate the cloud computing services, virtualized data center, emerging paradigm changes and executives of the largest independent provider ${ }^{[7]}$. VM virtualizations and migration capabilities to integrate their computer services and the minimum number of physical servers used to process the data center such as mining processing, security purpose, load balancing, server establishment, online maintenance, proactive fault tolerance and VM migration. Information's are use of the cloud computing to provide services for worldwide users. The consumer scientific controls the commercial domains hosting gives pervasive applications have costs and environmental contribution to the carbon footprint of data centers and cloud hosting applications that consume huge amounts of electrical energy. Therefore, reduces the environmental impact of cloud computing with the help of green computing and gossip protocol.

\subsection{Database}

Database systems serving cloud platforms must serve large numbers of applications. In addition to managing tolerant with small data footprints, different textures and shapes with variable load patterns such data platforms must minimize their operating costs by efficient resource sharing. The persistent database have the files are stored in network attached storage. VM migrate the database cache and the state of active transactions to ensure minimal impact on transaction execution while allowing transactions active during migration to continue executions and also guarantee the concurrency while ensuring correctness during failures ${ }^{[8]}$.

\subsection{Resource Allocation}

Dynamic Resource Management for Cloud Computing paradigm is an active area of research. The cost varies are considerably depending upon the configuration of resources by using them ${ }^{[6]}$. Efficient management of resources, cloud providers and users have the prime interests depending upon the size, capacity and flexibility for the cloud have been managing software which is able to use the hardware resources to succeed, and argued that the critical alone to provide the desired performance ${ }^{[5]}$. The successful resources management in the context of the resource constraints for the best private cloud solution, initial placement and load balancing when the resources to offers rich set. For example, during the peak of banking applications based on customer needs, they have number of servers that can be accessed from the cloud. In a moment have shut down the server and it can be used to save the energy.

\section{METHODOLOGY}

\subsection{Support Vector Machine}

The support vector machine (SVM) is a training algorithm for learning classification and regression rules of data ${ }^{[3]}$. The SVM is used to find the best classification functions to distinguish between members of the two classes in the trained data. The metrics for the best classification function can be realized geometrically and a linear classification function corresponds to a separating hyperplane $f(x)$ that passes through the middle and separating of the two classes. A function is determined the new data instance of $x_{n}$ can be classified by simply testing the sign of the function $f\left(x_{n}\right): x_{n}$ belongs to the positive class if $f\left(x_{n}\right)>0$.

\subsection{MD5 Method}

Step 1 - Append padded bits:

- The length of the messages 448 modulo of 512 that is similar to the modular padded.

- 64 bits to 512 bits long means they are just shy of being extended.

- The value of 1 bit is appended to the message and then the value 0 bit also appended so that the length in bits are equals 448 modulo 512.

Step 2 - Append length:

- 64 bit representation of $b$ is appended to the result of the previous step.

- Right that has a length of 512 bits of message.

Step 3 -Initialize MD Buffer

- A four-word buffer (A, B, C, D) is used to compute the message digest.

- Here every one has A, B, C, D is a 32-bit register

- Word A: 01234567 etc., the following hexadecimal values of these registers are initialized.

Step 4 -The 16 word blocks for process message.

- The input of three 32-bit words and getting the output of one 32-bit word four sub-functions.

$$
\mathrm{F}(\mathrm{X}, \mathrm{Y}, \mathrm{Z})=\mathrm{XY} v \operatorname{not}(\mathrm{X}) \mathrm{Z}
$$

The bits $X, Y$ and $Z$ are independent and also unbiased. The each bit of $F(X, Y, Z), G(X, Y, Z), H(X, Y, Z)$, and $I(X, Y, Z)$ will be independent and unbiased.

Step 5 -output

- Output A,B,C,D of the message digest.

- The ouput is start with the low order byte A and end with the high-order byte D.

\subsection{GREEN AND GOSSIP}

To initiate more environmental - friendly computing practices. There are some steps take toward a green computing strategy. Green Resource Allocator: It is act as the interface between 
the Cloud infrastructure and consumers. The interactions of the following components to support energy efficient resource management. The components are Arbitrator, OverhaulPrediction, Client Focused, Budget Processing, Power Consuming, Overhaul-Router, Storage Leadership and Gauge.

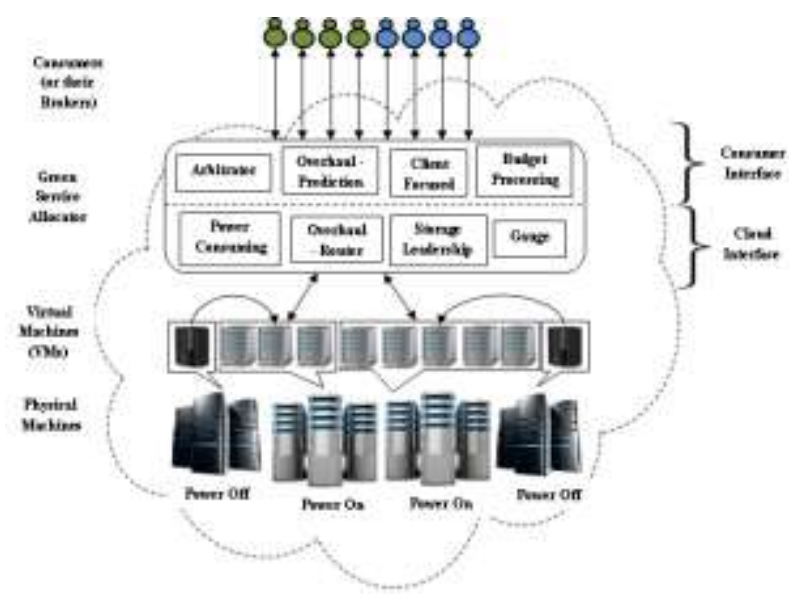

Figure: 3.1 Green Cloud Computing Architecture

Gossip huge clouds of green computing resource allocation based they aim to reduce server power consumption by integration with the specific objectives are sensible the resource allocation protocol and propose a common rumors. Under load the ethical and fair allocation of CPU resources to clients. The simulation result and the key performance of metrics for resource allocation process and suggest that it is the body has do not change with increasing size.

\section{SYSTEM IMPLEMENTATION}

\subsection{General}

In general, user interface design for consumer requests and getting response from the server. The consumer is valid provider means they are getting the further processes. It is used to interact with client and server for request and responding from the cloud storage.

\subsection{Implementation of Physical Machine}

In this module, to implement $\mathrm{n}$ number of physical server and which is interconnected with data storage. Each and every physical server have been separate identification like server IP, server port and server instance name. in this all the data stored in the storage device which the data can be applicable to source through physical server.

\subsection{Cloud Environment mining setup system}

It is provide the dynamic allocation of consumer request to the particular physical services and the physical server retrieve the consumers request based information from the storage device. The response of physical server is applicable for available data only. Cloud environment use the concept of SVM to populate the data from data centers.

\subsection{Highly Secure Client Processing}

In this system using MD5 based concept of achieving the securable data transmission between the consumer and the server. This MD5 is converted into the data normal format to undefined format. In this application apply the highly security for data transmission client request processing physical machine data population server mining user identification by server using the request name.

\subsection{Efficient Server Provisioning}

Dynamic allocation has been user request to the physical server in done by cloud environment by using the concept of protocol named as gossip ${ }^{[5]}$. This protocol is sufficient protocol for dynamic resource allocation and it gives response to the client at exact query matching server provisioning approach for minimized the cost, reduce the time and quick response. In order to assemble the cloud environmental setup and physical server storage device is very expensive but they are applying the mining setup. Show that it is must like expensive.

\section{EXPERIMENTAL RESULT}

The implementation of my concept is to create the $\mathrm{n}$ number of virtual machines and physical machines. In this machines have $\mathrm{n}$ number of information's are stored. This physical machine contains java connection based classes and service based concepts. In cloud environment system distributed set up of mined servers. The cloud server's shows all the information of the physical server in the data center.

In data center having mining setup for retrieve the data from the data storage. In data storage wants to store the number of files with the help of query processing from server. In this method using SVM for classify the data for the user query searched from the server.

The data center is the large storage network. The network wants to secure the stored information for the storage devices by using the cryptography technique. In this concept am using MD5 method for creating number of keys to secure the data from the storage devices. The valid user's are only views the information about the user queries. The key based concept is achieving the more secure for storage devices.

Finally, the server client communication is the very large process. In this having number of resources and it also intermediate process for file transfers to the user's and data storage. It is the nonstop processes so here using gossip protocol for green computing process. It is an automatically allocate the resources for file sharing in the data center.

\section{CONCLUSION}

To conclude that the concept have so many process in the cloud environment. The cloud environment has number of virtual machines and physical machines. These machines are used to store the number of data in the storage devices. The storage devices have been number of processes to mining the data.

In this the data can be find out from the storage device. The data retrieval by the user's from the cloud storage devices. In this the user given the request and get the response from the server. The SVM method is used for mined the data from the storage device. This method is used to classify the data and it is very efficient to gather some information without any unknown data.

The second process is to secure the data by using the method of MD5. The cryptographic technique is used to secure the information storage by using the key values. So the information storage is getting more secure without any leakage in the cloud environment.

The third process to find the delay provisioning for data center. The data center having so many request and response 
process for user's from the storage devices. In this device having continue processes so the machines want to overcome the delay processing by using the method of gossip protocol for green computing.

\section{REFERENCES}

[1] Qi Zhang, Mohamed Faten Zhani, Raouf Boutaba, Joseph L. Hellerstein "Dynamic Heterogeneity Aware Resource Provisioning in the Cloud" Proc.IEEE Trans. Cloud computing,2014.

[2] Jun Zhou, Xiaodong Lin, Xiaolei Dong, Zhenfu Cao "PSMPA: Patient Self-controllable and Multi-level Privacy-preserving Cooperative Authentication in Distributed m-Healthcare Cloud Computing System" Proc. IEEE Tans. Parallel and Distributed System, 2014.

[3] Lu Zhang and Xueyan Tang "The Client Assignment Problem for Continuous Distributed Interactive Applications: Analysis, Algorithms, and Evaluation" Proc. IEEE Trans. Parallel and Distributed System, 2014.

\section{Authors}

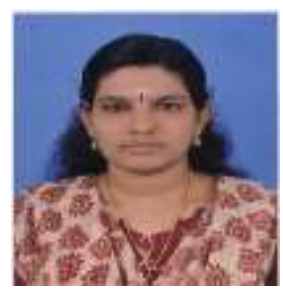

MS. Anju Aravind K, received the Bachelor of Engineering in Anna University, TamilNadu, India in 2011. PG Scholar Currently persuing her M.E CSE degree in shree Venkateshwara Hi-Tech Engg College, Gobi, TamilNadu, India.

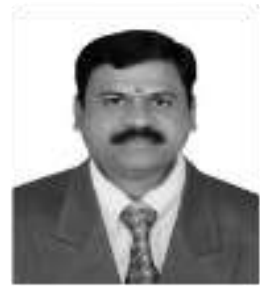

Dr.T.Senthil Prakash received the Ph.D. degree from the PRIST University, Thanjavur, India in 2013 and M.E(CSE) degree from Vinayaka Mission's University, Salem, India in 2007 and M.Phil.,MCA.,B.Sc(CS) degrees from Bharathiyar University, Coimbatore India, in 2000,2003 and 2006 respectively, all in Computer Science and Engineering. He is a Member in ISTE New Delhi, India, IAENG, Hong Kong..IACSIT, Singapore SDIWC, USA. He has the experience in Teaching of $10+$ Years and in Industry 2 Years. Now He is currently working as a Professor and Head of the Department of Computer Science and Engineering in Shree Venkateshwara Hi-Tech Engineering College, Gobi, Tamil Nadu, and India. His research interests include Data Mining, Data Bases, Artificial Intelligence, Software Engineering etc.,He has published several papers in 17 International Journals, 43 International and National Conferences.

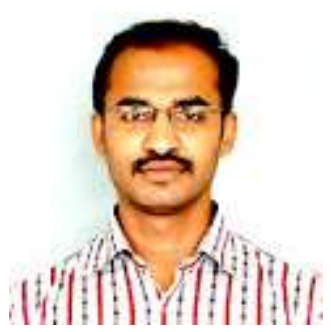

Mr.M.Rajesh, Received the Bachelor of Engineering in Anna University, TamilNadu, India in 2007 and Master of Engineering from Kongu Engineering College of India in 2012. Currently he is doing Ph.D at Bharath University, Chennai. His research interests include cloud computing in resource provisioning. 